\title{
Exploring scalar field dynamics with Gaussian processes
}

\author{
Remya Nair, ${ }^{a}$ Sanjay Jhingan ${ }^{a}$ and Deepak Jain ${ }^{b}$ \\ ${ }^{a}$ Centre for Theoretical Physics, \\ Jamia Millia Islamia, New Delhi 110025, India \\ ${ }^{b}$ Deen Dayal Upadhyaya College, \\ University of Delhi, New Delhi 110015, India \\ E-mail: remya_phy@yahoo.com, sanjay.jhingan@gmail.com,djain@ddu.du.ac.in
}

\begin{abstract}
The origin of the accelerated expansion of the Universe remains an unsolved mystery in Cosmology. In this work we consider a spatially flat Friedmann-Robertson-Walker (FRW) Universe with non-relativistic matter and a single scalar field contributing to the energy density of the Universe. Properties of this scalar field, like potential, kinetic energy, equation of state etc. are reconstructed from Supernovae and BAO data using Gaussian processes. We also reconstruct energy conditions and kinematic variables of expansion, such as the jerk and the slow roll parameter. We find that the reconstructed scalar field variables and the kinematic quantities are consistent with a flat $\Lambda \mathrm{CDM}$ Universe. Further we find that the null energy condition is satisfied for the redshift range of the Supernovae data considered in the paper but, the strong energy condition is violated.
\end{abstract}

Keywords: Cosmic acceleration, Supernovae, Baryon Acoustic Oscillations, Gaussian processes 


\section{Contents}

1 Introduction 1

2 Methodology 3

2.1 Dark energy parameters 3

2.2 Gaussian Processes $\quad 5$

2.3 Data sets $\quad 8$

3 Results and discussion $r$

3.1 Dark energy dynamics 12

3.2 Kinematic variables: $j$ and $\epsilon_{H} \quad 13$

\section{Introduction}

Providing an explanation for the observed accelerated expansion of the Universe, is one of the bigger challenges in Cosmology today. The evidence for existence of dark energy (DE) which is believed to source this acceleration [1-3], has been accumulating ever since its first hints from supernovae (SNeIa) observations [4]. Today there are multiple probes all substantiating the idea that the Universe is accelerating [5]. However, even in this era of precision Cosmology, we know little about this unknown form of energy. Many ambitious projects are being carried out and others are lined up for the future with the goal of better understanding the nature of DE [6]. On the theoretical side, the simplest and one of the most successful DE candidate is the cosmological constant $\Lambda$, but it suffers from serious theoretical problems (fine tuning and coincidence) [7]. If interpreted as a fluid with pressure and energy density, the cosmological constant has an equation of state (eos) $\omega=-1$. Observations constrain the eos of DE to be near -1 today, but the time variation in $\omega$ is still unconstrained. An alternative to the cosmological constant are variable DE models. A variety of scalar field models have been proposed in literature: quintessence, K-essence, Tachyon etc. [3]. All these models are constructed within the framework of general relativity (GR). A modification of gravity at large scales is also considered as a possible explanation for the late time acceleration of the Universe (for a review see [8] and references therein), but in this paper we work within the framework of GR.

Various methods have been employed to understand the nature of DE by estimating DE parameters from observations. The most frequently used model independent method is the parametric approach. A standard way to model possible departure from the cosmological constant, is to parametrise the eos parameter $\omega(z)$ to account for a value different from -1 (i.e. a possible variation with redshift). One can either assume $\omega=$ constant or allow for specific redshift variations, for example $\omega(z)=\omega_{0}+\omega_{1} z /(1+z)$, where $\omega_{0}$ and $\omega_{1}$ are constants. Although this is a simple way to incorporate the dynamical aspects of DE and quantify its properties with a few numbers, the results are strongly dependent on the parametrization chosen. For example, Bassett et al., have shown that the confidence intervals inferred from standard two-parameter expansions of the eos are typically untrustworthy [9]. Another way to understand DE behaviour is to analyse kinematic variables like the Hubble rate $H(z)$, the deceleration parameter $q(z)$ or the jerk parameter $j(z)$, which are all constructed from the derivatives of the scale factor $a(z)$. There have been attempts to constrain $H_{0}, q_{0}$ and $j_{0}$ (the 
value of these parameters at $z=0$ ), by analysing the cosmographic expansion of the luminosity distance [10]. The kinematic approach is advantageous since one does not need to assume any specific composition of the Universe and there have been attempts to reconstruct the cosmic expansion history by parametrising $q(z)$ or $j(z)$ [11]. Also worth mentioning are the efforts to study the geometric expansion history of the Universe, by Sahni and collaborators. They proposed the use of diagnostic parameters called 'statefinders', constructed from the scale factor of the Universe and its time derivatives [12]. It is expected that future supernovae measurements would have the constraining power to differentiate between different forms of dark energy using these diagnostic parameters. In a related work, Sahni et al., proposed the Om diagnostic which is a combination of the Hubble parameter and the cosmological redshift and provides a null test of dark energy being a cosmological constant [13]. This work was further extended by Shafieloo et al., who proposed the Om3 diagnostic [14]. This is designed for baryon acoustic oscillations (BAO) data and can combine information from BAO and type Ia supernovae (SNeIa) to give another null diagnostic of the cosmological constant.

As mentioned earlier, parametrisation of DE variables can introduce bias in a analysis and non-parametric techniques may offer a solution to these problems. Here one does not need to assume an ad-hoc functional form for the quantities of interest $(\omega(z), H(z), q(z)$ or $j(z)$ etc.), which could bias the results if the actual form of the evolution is different from the one assumed. In this regard various non-parametric methods have been proposed, which use uncorrelated redshift binning, Bayesian treatment based on maximum entropy methods etc. to reconstruct the expansion history and DE eos [15, 16]. Principal Component Analysis (PCA) is another (now) commonly used non-parametric method. Huterer and Starkman were among the first to advocate the use of PCA to analyse DE properties [17]. Shapiro and Turner used PCA to show that there is strong evidence from the Riess supernovae data that the Universe once accelerated and that the deceleration parameter was higher in the past [18] and Dick et al., presented a method for reducing the cosmological data to constraints on the mode amplitudes of the dark energy density [19] using PCA. But it is important to remember that this technique has caveats. Since most of the information is contained in the first few eigenmodes, one makes a truncation and keeps the best constrained principal components for further analysis. Keeping all the eigenmodes would amount to zero information loss, but the error bars would be too large to give any meaningful estimates. The process of truncation can potentially lead to biases and effect the estimated errors on the modes. Kitching and Amara showed that the eigen-decomposition of the Fisher matrix (the main step of PCA) is sensitive to both the order of expansion and the initial basis set used in the construction of the Fisher matrix [20]. Putter and Linder further gave some quantified examples to argue that oversimplifying PCA interpretation can lead to misinterpretations [21].

Another non-parametric method, the Smoothing technique developed by Shafieloo et al., uses Gaussian kernel to smooth the data (in their case SNeIa data) to calculate cosmological functions like $H(z)$ and $\omega(z)$ [22]. The technique can also be used to distinguish between $\Lambda \mathrm{CDM}$ and evolving dark energy models. Another technique, developed by Shafieloo, Clifton and Ferreira, the 'Crossing statistics' can be used even in cases where the intrinsic dispersion of a data set is not well known, and can distinguish between different models in cases where the standard $\chi^{2}$ statistic fails [23]. In a recent paper Arman Shafieloo showed that these techniques can be combined to reconstruct the expansion history of the Universe without putting any prior on the cosmological quantities such as the eos [24]. Also worth mentioning is recent work by Nesseris and Bellido who proposed a method for model independent and bias-free reconstruction of various cosmological distances using Genetic algorithm [25]. They 
further showed that the method can be used to estimate the errors on the reconstructed quantities analytically and their estimates agree with the standard error estimation technique involving Fisher matrix.

An alternative non-parametric approach which has garnered attention lately is the Gaussian Process modelling (GP) which is the basis of this paper and is discussed in detail in the next section. GP is a non-parametric regression approach based on a generalization of the Gaussian probability distribution. Many attempts have been made to understand DE dynamics through GP methodology. GP has been used to reconstruct the eos of DE and to reconstruct the history of the expansion rate and deceleration parameter as a function of redshift [26-28]. In a related work, similar in spirit to GP, Crittenden et al. developed a non-parametric Bayesian method for reconstructing the time evolution of the DE eos by choosing a prior which minimises variance and bias in the reconstruction [29].

As mentioned earlier, one of the problems faced by the cosmological constant is the coincidence problem (why DE dominates today?). It can be resolved by allowing the DE to be dynamical. Most of the observational data is consistent with a $\Lambda$ cold dark matter $(\Lambda \mathrm{CDM})$ Universe, and hence any viable DE model has to mimic the cosmological constant today. Since there is no theoretical compulsion for the DE density to be constant, and observations today cannot rule out dynamical models for DE, there is a plethora of models which are proposed as an alternative to the cosmological constant (for a review on dark energy reconstruction see [2]). In the presence of so many theoretical models for DE, it becomes important to constrain the possible shape of the DE potential to distinguish between different models. In this regard, there have been many attempts to reconstruct DE properties. Saini et al., proposed a model-independent method, based on a versatile analytical form for the luminosity distance for estimating the form of the potential of the scalar field driving the cosmic acceleration, and the associated eos [30]. There have been several other attempts to reconstruct the scalar potential and eos from observational data, see [31] for examples.

The aim of this paper is to reconstruct and constrain the properties of the scalar field $\phi$, which is assumed to contribute to the energy density of the Universe along with nonrelativistic matter. We also analyse energy conditions and two kinematic parameters, the 'jerk' and the 'slow roll'. We consider a spatially flat FRW Universe since it agrees well with the observations today. In section 2, we outline the main formulae used for the reconstruction (2.1), discuss the GP methodology (2.2) and give details of the data sets used (2.3) for reconstruction. The results are presented and discussed in section 3.

\section{Methodology}

\subsection{Dark energy parameters}

Scalar field dynamics. We assume a flat FRW Universe with energy contribution coming from non-relativistic matter and a single scalar field. The equation governing the evolution of this scalar field is

$$
\ddot{\phi}+3 H \dot{\phi}=-\frac{\partial V}{\partial \phi},
$$

and the pressure and density of the scalar field (assuming it is spatially homogeneous) is 
given by

$$
\begin{aligned}
& P_{\phi}=\frac{\dot{\phi}^{2}}{2}-V(\phi), \\
& \rho_{\phi}=\frac{\dot{\phi}^{2}}{2}+V(\phi) .
\end{aligned}
$$

The Friedmann equations in this case reduce to

$$
\begin{aligned}
H^{2} & =\frac{8 \pi G}{3}\left(\rho_{m}+\rho_{\phi}\right), \\
\ddot{a} & =-\frac{4 \pi G}{3}\left(\rho_{m}+\rho_{\phi}+3 P_{\phi}\right) .
\end{aligned}
$$

Re-arranging the above two sets of equations we get,

$$
\begin{aligned}
\frac{8 \pi G}{3 H_{0}^{2}} V(z) & =\frac{H^{2}}{H_{0}^{2}}-\frac{1+z}{3 H_{0}^{2}} H H^{\prime}-\frac{\Omega_{m}(1+z)^{3}}{2}, \\
\frac{8 \pi G}{3 H_{0}^{2}} \dot{\phi}^{2} & =\frac{2(1+z)}{3 H_{0}^{2}} H H^{\prime}-\Omega_{m}(1+z)^{3} .
\end{aligned}
$$

Here, an over-dot indicates differentiation with respect to time, and prime indicates differentiation with respect to redshift $z$. Similarly, one can reconstruct the eos of the scalar field $\omega\left(=P_{\phi} / \rho_{\phi}\right)$, using these expressions. Recent constraints on a constant eos from Planck collaboration is $\omega=-1.09 \pm 0.17$ (Planck+WMAP+Union2.1) and on a two parameter model of eos $\omega(z)=\omega_{0}+\omega_{a}(1-a)$ is $\omega_{0}=-1.04_{-0.69}^{+0.72}$ and $\omega_{a}<1.32$ (all error bars are at $95 \%$ confidence) [32].

The evolution of these scalar field variables $\left(V, \dot{\phi}^{2}, \omega\right)$ can be estimated, if one can reconstruct the Hubble parameter and its derivatives from observations. Now, in a flat FRW Universe the luminosity distance $D_{L}(z)$, can be written as

$$
D_{L}(z)=\frac{c(1+z)}{H_{0}} \int_{0}^{z} \frac{d z^{\prime}}{\mathcal{H}\left(z^{\prime}\right)}
$$

where $c$ is the speed of light, $H_{0}=H(z=0)$ and $\mathcal{H}(z)=H(z) / H_{0}$. Since the Hubble parameter can be written in terms of the derivative of the distance, we can also derive scalar field variables in terms of $D_{L}(z)$ (and its derivatives).

Energy conditions. In general relativity (GR), "energy conditions" is a way of implementing the experimentally observed notion of positivity of energy density. Different energy conditions impose restrictions on various linear combinations of energy momentum tensor. The powerful theorems proven in GR, the singularity theorems, the positive energy theorem etc., all assume some notion of positivity of energy density implemented through energy conditions. These conditions were introduced in Hawking and Ellis [33] as coordinate independent inequalities on energy momentum tensor in Einstein field equations. Because of their simplicity and model independence they are widely discussed in GR [34]. Various energy conditions (null energy condition (NEC), weak energy condition (WEC), strong energy condition (SEC), and dominant energy condition (DEC)), in the setting of FRW background 
can be expressed as constraints on energy density and pressure as [35]

$$
\begin{aligned}
& N E C: \quad \rho+p \geq 0, \\
& W E C: \quad \rho+p \geq 0, \quad \text { and } \rho \geq 0 \\
& S E C: \quad \rho+p \geq 0, \quad \text { and } \rho+3 p \geq 0 \\
& D E C: \quad \rho \geq 0, \quad \text { and } \quad-\rho \leq p \leq p
\end{aligned}
$$

The energy conditions and their cosmological relevance was first explored in detail by Matt Visser [36]. Later on, various authors used observational data to study the violations of the energy conditions and their consequences [37]. Thus energy conditions play an important role in imposing model independent bounds on some of the key properties of DE. In this paper we explore the validity of the null and strong energy conditions (NEC \& SEC). The SEC implies that the expansion of the Universe is slowing down, hence violation of SEC $(\ddot{a}>0)$ means accelerated expansion. The violation of SEC also implies $\omega \equiv P / \rho<-1 / 3$.

Kinematic parameters. In the absence of a compelling model to describe the cosmic acceleration, it is useful to study kinematic parameters derived from the derivatives of the scale factor $a$. The Hubble parameter $H(t)=\dot{a} / a$, the deceleration parameter $q(t)=-a \ddot{a} / \dot{a}^{2}$, and the jerk parameter $j(t)=\dddot{a} a^{2} / a^{3}$, are such kinematic parameters and are all theoretically equivalent since one can be written in terms of the derivative or integral of the other. The jerk parameter is favored sometimes as a good kinematic choice, since for a $\Lambda$ CDM Universe $j(z)=1$, for all $z$. Hence it can be used to distinguish different DE models and there have been several attempts to constrain the jerk parameter using observational data [38-40]. $j$ can be expressed in terms of the Hubble parameter as:

$$
j=1+\frac{(1+z)^{2} H^{\prime \prime}(z)}{H(z)}-\frac{2(1+z) H^{\prime}(z)}{H(z)}+\frac{(1+z)^{2} H^{\prime}(z)^{2}}{H(z)^{2}} .
$$

The Slow roll parameter $\epsilon_{H}=-\ddot{a} / a H^{2}+1$ is another parameter employed to understand the dynamics of expansion due to the scalar field and can be used to check the presence of an inflationary phase $\left(\epsilon_{H}<<1\right)$ in the Universe. Note that in the case of late time DE dynamics this parameter is not entirely reliable as a scalar field parameter, since the density of matter is not negligible. But one can define this parameter in terms of the derivative of the Hubble parameter and use it as a measure to check the existence of an accelerated expansion [3]. $\epsilon_{H}<1$ is equivalent to the accelerated expansion $\ddot{a}>0$, and $\epsilon_{H}>1$ is the decelerated expansion $\ddot{a}<0$, where

$$
\epsilon_{H}=(1+z) \frac{H^{\prime}(z)}{H(z)} .
$$

Under the slow roll limit $\left(\epsilon_{H}<<1\right)$ the Hubble parameter becomes almost constant and the Universe expands as if it is dominated by the cosmological constant.

\subsection{Gaussian Processes}

A Gaussian Process (GP) is a collection of random variables, any finite number of which have a joint Gaussian distribution [41]. Similar to a Gaussian distribution, which is a distribution of a random variable characterized by a mean and a covariance, a GP is a distribution over functions, characterized by a mean function and a covariance matrix. Given a set of observations the aim is to infer the relation between independent and dependent variables. 
In parametric regression one assumes some functional dependence of the output on the input $f(x, \boldsymbol{\theta})$, where $\boldsymbol{\theta}$ represents the set of model parameters. Here regression entails finding the values of $\boldsymbol{\theta}$ which best describe the data. The best fit parameters are usually found by minimizing a chi-squared merit function. Similarly, in GP modelling the function $f(x)$ is represented as

$$
f(x) \sim G P\left(\mu(x), k\left(x, x^{\prime}\right)\right),
$$

i.e, the value of $f(x)$ at any point $x$, is a Gaussian random variable with mean $\mu(x)$ and covariance $k\left(x, x^{\prime}\right)$ :

$$
\begin{aligned}
\mu(x) & =E(f(x)), \\
k\left(x, x^{\prime}\right) & =E\left((f(x)-\mu(x))\left(f\left(x^{\prime}\right)-\mu\left(x^{\prime}\right)\right)\right) .
\end{aligned}
$$

A number of possible choices for the covariance functions exist: squared exponential, polynomial, spline etc. By choosing a particular covariance function, we choose a basis of the function space to expand the target function. Here we chose the commonly used squared exponential function for its simplicity and also because it is infinitely differentiable, a property which is useful while constructing higher order derivatives. The squared exponential covariance function is expressed as:

$$
k\left(x, x^{\prime}\right)=\sigma_{f}^{2} \exp \left(-\frac{\left(x-x^{\prime}\right)^{2}}{2 l^{2}}\right) .
$$

This functions is parameterized by the two parameters, $\sigma_{f}$ and $l$ (known as hyperparameters). These parameters represent the length scales in the GP. $l$ corresponds to the correlation length along which the successive $f(x)$ values are correlated and $\sigma_{f}$ controls the variation in $f(x)$ relative to the mean of the process. Thus, the covariance between output variables are written as a function of the inputs. One can see that the covariance is maximum for variables whose inputs are very close. This is something we do expect for smooth functions. The matrix elements of the covariance matrix for the GP: $K(\mathbf{X}, \mathbf{X})$, are given by

$$
[\mathbf{K}(\mathbf{X}, \mathbf{X})]_{i, j}=k\left(x_{i}, x_{j}\right)
$$

One can generate random functions using this covariance matrix even without any data. Similar to the function $f(x)$, the data $\mathbf{y}$ can be represented using GP:

$$
y \sim G P\left(\mu(x), k\left(x, x^{\prime}\right)\right),
$$

Given a set of inputs $\mathbf{X}$ (also called training vectors), outputs $\mathbf{y}$ (the data set, also called target) and the covariance matrix $\mathbf{K}(\mathbf{X}, \mathbf{X})$, our aim is to make inference about the function $f(x)$ at some other points $\hat{\mathbf{X}}$. The joint probability distribution for data $y$ and reconstructed function $\hat{f}$ is given by

$$
\left(\begin{array}{l}
\mathbf{y} \\
\hat{\mathbf{f}}
\end{array}\right) \sim N\left(\left[\begin{array}{l}
\boldsymbol{\mu} \\
\hat{\boldsymbol{\mu}}
\end{array}\right],\left[\begin{array}{rr}
\mathbf{K}(\mathbf{X}, \mathbf{X})+\mathbf{C} & \mathbf{K}(\mathbf{X}, \hat{\mathbf{X}}) \\
\mathbf{K}(\hat{\mathbf{X}}, \mathbf{X}) & \mathbf{K}(\hat{\mathbf{X}}, \hat{\mathbf{X}})
\end{array}\right]\right),
$$

where $\boldsymbol{\mu}$ and $\hat{\boldsymbol{\mu}}$ are the assumed means (initial guess) and $\mathbf{C}$ is the covariance matrix of the data. If the data points are uncorrelated then $\mathbf{C}$ is a diagonal matrix. Say, $p$ and $q$ are the number of points in $\mathbf{X}$ and $\hat{\mathbf{X}}$ respectively, and the joint covariance matrix

$$
\boldsymbol{\Sigma}=\left[\begin{array}{cc}
\mathbf{K}(\mathbf{X}, \mathbf{X})+\mathbf{C}, \mathbf{K}(\mathbf{X}, \hat{\mathbf{X}}) \\
\mathbf{K}(\hat{\mathbf{X}}, \mathbf{X}), & \mathbf{K}(\hat{\mathbf{X}}, \hat{\mathbf{X}})
\end{array}\right]=\left[\begin{array}{ll}
\boldsymbol{\Sigma}_{11} & \boldsymbol{\Sigma}_{12} \\
\boldsymbol{\Sigma}_{21} & \boldsymbol{\Sigma}_{22}
\end{array}\right],
$$


then one can write

$$
P(\mathbf{y}, \hat{\mathbf{f}})=\frac{1}{(2 \pi)^{(p+q) / 2}|\boldsymbol{\Sigma}|^{1 / 2}} \exp \left\{\left[(\mathbf{y}-\boldsymbol{\mu})^{T},(\hat{\mathbf{f}}-\hat{\boldsymbol{\mu}})^{T}\right] \boldsymbol{\Sigma}^{-1}\left[\begin{array}{l}
\mathbf{y}-\boldsymbol{\mu} \\
\hat{\mathbf{f}}-\hat{\boldsymbol{\mu}}
\end{array}\right]\right\}
$$

The matrix elements of the inverse of the joint covariance matrix $\boldsymbol{\Sigma}$ can be evaluated as follows

$$
\boldsymbol{\Sigma}^{-1}=\left[\begin{array}{cc}
\mathbf{K}(\mathbf{X}, \mathbf{X})+\mathbf{C}, \mathbf{K}(\mathbf{X}, \hat{\mathbf{X}}) \\
\mathbf{K}(\hat{\mathbf{X}}, \mathbf{X}), & \mathbf{K}(\hat{\mathbf{X}}, \hat{\mathbf{X}})
\end{array}\right]^{-1}=\left[\begin{array}{ll}
\boldsymbol{\Sigma}^{11} & \boldsymbol{\Sigma}^{12} \\
\boldsymbol{\Sigma}^{21} & \boldsymbol{\Sigma}^{22}
\end{array}\right]
$$

where

$$
\begin{gathered}
\boldsymbol{\Sigma}^{11}=(\mathbf{K}(\mathbf{X}, \mathbf{X})+\mathbf{C})^{-1}+(\mathbf{K}(\mathbf{X}, \mathbf{X})+\mathbf{C})^{-1} \mathbf{K}(\mathbf{X}, \hat{\mathbf{X}}) \\
{\left[\mathbf{K}(\hat{\mathbf{X}}, \hat{\mathbf{X}})-\mathbf{K}(\mathbf{X}, \hat{\mathbf{X}})^{T}(\mathbf{K}(\mathbf{X}, \mathbf{X})+\mathbf{C})^{-1} \mathbf{K}(\mathbf{X}, \hat{\mathbf{X}})\right]^{-1}} \\
\mathbf{K}(\mathbf{X}, \hat{\mathbf{X}})^{T}(\mathbf{K}(\mathbf{X}, \mathbf{X})+\mathbf{C})^{-1} \\
\boldsymbol{\Sigma}^{22}=\mathbf{K}(\hat{\mathbf{X}}, \hat{\mathbf{X}})^{-1}+\mathbf{K}(\hat{\mathbf{X}}, \hat{\mathbf{X}})^{-1} \mathbf{K}(\mathbf{X}, \hat{\mathbf{X}})^{T} \\
{\left[\mathbf{K}(\mathbf{X}, \mathbf{X})+\mathbf{C}-\mathbf{K}(\mathbf{X}, \hat{\mathbf{X}}) \mathbf{K}(\hat{\mathbf{X}}, \hat{\mathbf{X}})^{-1} \mathbf{K}(\mathbf{X}, \hat{\mathbf{X}})^{T}\right]^{-1}} \\
\mathbf{K}(\mathbf{X}, \hat{\mathbf{X}}) \mathbf{K}(\hat{\mathbf{X}}, \hat{\mathbf{X}})^{-1} \\
\boldsymbol{\Sigma}^{12}=-(\mathbf{K}(\mathbf{X}, \mathbf{X})+\mathbf{C})^{-1} \mathbf{K}(\mathbf{X}, \hat{\mathbf{X}})\left[\mathbf{K}(\hat{\mathbf{X}}, \hat{\mathbf{X}})-\mathbf{K}(\mathbf{X}, \hat{\mathbf{X}})^{T}-\right. \\
\left.(\mathbf{K}(\mathbf{X}, \mathbf{X})+\mathbf{C})^{-1} \mathbf{K}(\mathbf{X}, \hat{\mathbf{X}})\right]^{-1}
\end{gathered}
$$

Further the determinant of the matrix $\boldsymbol{\Sigma}$ is given by

$$
\operatorname{det}(\boldsymbol{\Sigma})=\operatorname{det}\left(\boldsymbol{\Sigma}_{11}\right) \operatorname{det}\left(\boldsymbol{\Sigma}_{22}-\boldsymbol{\Sigma}_{12}^{T} \boldsymbol{\Sigma}_{11}^{-1} \boldsymbol{\Sigma}_{12}\right)
$$

Substituting these expressions for $\boldsymbol{\Sigma}^{11}, \boldsymbol{\Sigma}^{12} \& \boldsymbol{\Sigma}^{22}$ we obtain the joint probability distribution for data $\mathbf{y}$ and the reconstructed function $\hat{\mathbf{f}}$ as

$$
\begin{aligned}
& P(\mathbf{y}, \hat{\mathbf{f}})=\frac{1}{(2 \pi)^{p / 2}\left|\boldsymbol{\Sigma}_{11}\right|^{1 / 2}} \exp \left[-\frac{1}{2}(\mathbf{y}-\boldsymbol{\mu})^{T} \boldsymbol{\Sigma}_{11}^{-1}(\mathbf{y}-\boldsymbol{\mu})\right] \\
& \frac{1}{(2 \pi)^{q / 2}|\mathbf{A}|^{1 / 2}} \exp \left[-\frac{1}{2}(\hat{\mathbf{f}}-\mathbf{a})^{T} \mathbf{A}^{-1}(\hat{\mathbf{f}}-\mathbf{a})\right],
\end{aligned}
$$

where

$$
\mathbf{a}=\hat{\boldsymbol{\mu}}+\mathbf{K}(\mathbf{X}, \hat{\mathbf{X}})^{T}(\mathbf{K}(\mathbf{X}, \mathbf{X})+\mathbf{C})^{-1}(\mathbf{y}-\boldsymbol{\mu})
$$

and

$$
\mathbf{A}=\mathbf{K}(\hat{\mathbf{X}}, \hat{\mathbf{X}})-\mathbf{K}(\mathbf{X}, \hat{\mathbf{X}})^{T}(\mathbf{K}(\mathbf{X}, \mathbf{X})+\mathbf{C})^{-1} \mathbf{K}(\mathbf{X}, \hat{\mathbf{X}})
$$

The marginal distribution of $\mathbf{y}$ is given by

$$
P(\mathbf{y})=\int P(\mathbf{y}, \hat{\mathbf{f}}) d \hat{\mathbf{f}}=\frac{1}{(2 \pi)^{p / 2}\left|\boldsymbol{\Sigma}_{11}\right|^{1 / 2}} \exp \left[-\frac{1}{2}(\mathbf{y}-\boldsymbol{\mu})^{T} \boldsymbol{\Sigma}_{11}^{-1}(\mathbf{y}-\boldsymbol{\mu})\right],
$$

and the conditional distribution $P(\hat{\mathbf{f}} \mid \mathbf{y})$ is

$$
P(\hat{\mathbf{f}} \mid \mathbf{y})=\frac{P(\mathbf{y}, \hat{\mathbf{f}})}{P(\mathbf{y})}=\frac{1}{(2 \pi)^{q / 2}|\mathbf{A}|^{1 / 2}} \exp \left[-\frac{1}{2}(\hat{\mathbf{f}}-\mathbf{a})^{T} \mathbf{A}^{-1}(\hat{\mathbf{f}}-\mathbf{a})\right],
$$


i.e, the reconstructed function $\hat{\mathbf{f}}(\hat{\mathbf{X}})$ has a Gaussian normal distribution given by

$$
\hat{\mathbf{f}} \sim G P(\mathbf{a}, \mathbf{A}) .
$$

Note that $\sigma_{f}$ and $l$ are unknown parameters of the GP. The training of a GP involves selecting these parameters. This can be done by maximising the marginal log-likelihood probability $\ln P(y)$ (from 2.18)

$$
\ln P(\mathbf{y})=-\frac{1}{2}(\mathbf{y}-\boldsymbol{\mu})^{T}[\mathbf{K}(\mathbf{X}, \mathbf{X})+\mathbf{C}]^{-1}(\mathbf{y}-\boldsymbol{\mu})-\frac{1}{2} \ln |\mathbf{K}(\mathbf{X}, \mathbf{X})+\mathbf{C}|-\frac{p}{2} \ln 2 \pi
$$

This is an approximation, and can be used if the posterior for $\boldsymbol{\theta}$ (which includes any other parameters that the analysis may have), is fairly well peaked. If there are many parameters in the analysis (which include those coming from an assumed mean function), this kind of optimization may result in over-fitting. In our analysis, we sample the hyperparameter space and the probability distributions of the reconstructed function are weighted by the posterior distributions of the hyperparameters. We also need to reconstruct derivative function for our analysis. The derivative of a GP is another GP and hence, GP modelling can be used to reconstruct the derivatives as well. If $k\left(x_{i}, x_{j}\right)$ is the covariance between function values then the covariance between function and its derivatives, and between derivatives can be obtained by differentiating the original covariance function

$$
\operatorname{cov}\left(f_{i}, \frac{\partial f_{j}}{\partial x_{j}}\right)=\frac{\partial k\left(x_{i}, x_{j}\right)}{\partial x_{j}}, \operatorname{cov}\left(\frac{\partial f_{i}}{\partial x_{i}}, \frac{\partial f_{j}}{\partial x_{j}}\right)=\frac{\partial^{2} k\left(x_{i}, x_{j}\right)}{\partial x_{i} \partial x_{j}},
$$

and one can evaluate the covariance matrices for the derivatives:

$\left[\mathbf{K}^{\prime}(\mathbf{X}, \hat{\mathbf{X}})\right]_{i, j}=\partial k\left(x_{i}, \hat{x}_{j}\right) / \partial \hat{x}_{j},\left[\mathbf{K}^{\prime \prime}(\hat{\mathbf{X}}, \hat{\mathbf{X}})\right]_{i, j}=\partial^{2} k\left(\hat{x}_{i}, \hat{x}_{j}\right) / \partial \hat{x}_{i} \partial \hat{x}_{j}$ etc.

As before, the mean value and the covariance for the reconstructed derivative function can be obtained from the conditional distribution $P\left(\hat{\mathbf{f}}^{\prime} \mid \mathbf{y}\right)$ as follows (and higher order derivatives can be constructed similarly),

$$
\overline{\hat{f}}^{\prime}=\hat{\boldsymbol{\mu}}^{\prime}+\mathbf{K}^{\prime}(\mathbf{X}, \hat{\mathbf{X}})^{T}(\mathbf{K}(\mathbf{X}, \mathbf{X})+\mathbf{C})^{-1}(\mathbf{y}-\boldsymbol{\mu})
$$

and

$$
\operatorname{cov}\left(\hat{\mathbf{f}}^{\prime}\right)=\mathbf{K}^{\prime \prime}(\hat{\mathbf{X}}, \hat{\mathbf{X}})-\mathbf{K}^{\prime}(\mathbf{X}, \hat{\mathbf{X}})^{T}(\mathbf{K}(\mathbf{X}, \mathbf{X})+\mathbf{C})^{-1} \mathbf{K}^{\prime}(\mathbf{X}, \hat{\mathbf{X}}) .
$$

To construct functions that depend on combinations of these reconstructed functions i.e. $\hat{\mathbf{f}}$, $\hat{\mathbf{f}}^{\prime} \hat{\mathbf{f}}^{\prime \prime}$ etc., one samples from the joint probability distributions of these functions.

\subsection{Data sets}

For our analysis we used distance modulus data from SNeIa and Hubble measurements from BAO data. The priors on all the hyperparameters are assumed to be flat, and we give rather broad prior ranges as specified below for each data set. The details of the data sets used are given below:

BAO data. We use eight $H(z)$ measurements from BAO data compiled from different groups in the redshift range $0.24<z<2.3$. Gaztanaga et al., used the spectroscopic Sloan Digital Sky Survey (SDSS) data to give a direct measurement of the Hubble parameter $H(z)$ as a function of redshift at $z=0.24 \& 0.43$ [42]. Chuang and Wang used the sample of luminous red galaxies from the SDSS Data Release 7 to give Hubble measurement at 


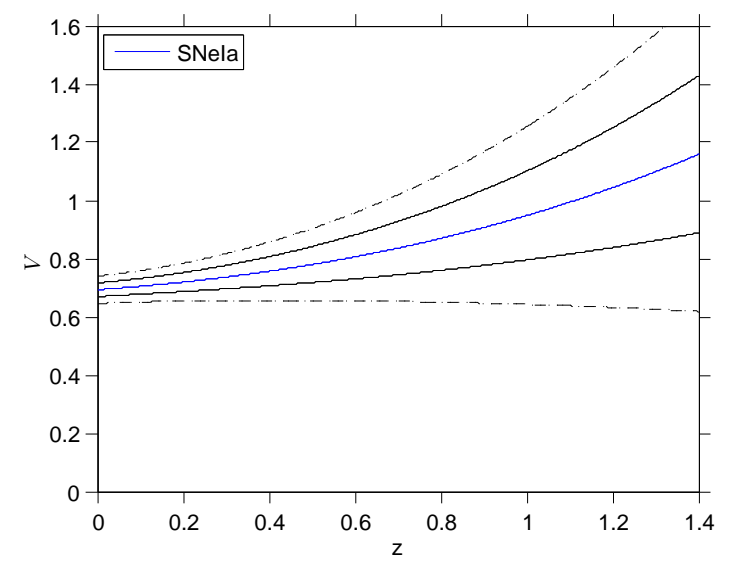

(a)

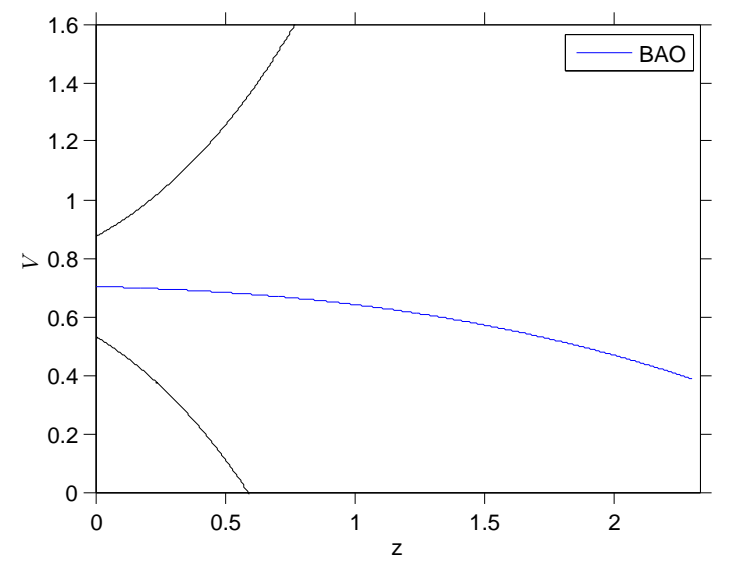

(b)

Figure 1: Solid lines show the reconstructed potential as a function of redshift with $1 \sigma$ error bars (black curves). In figure (a) the dashed lines corresponds $2 \sigma$ error bars.

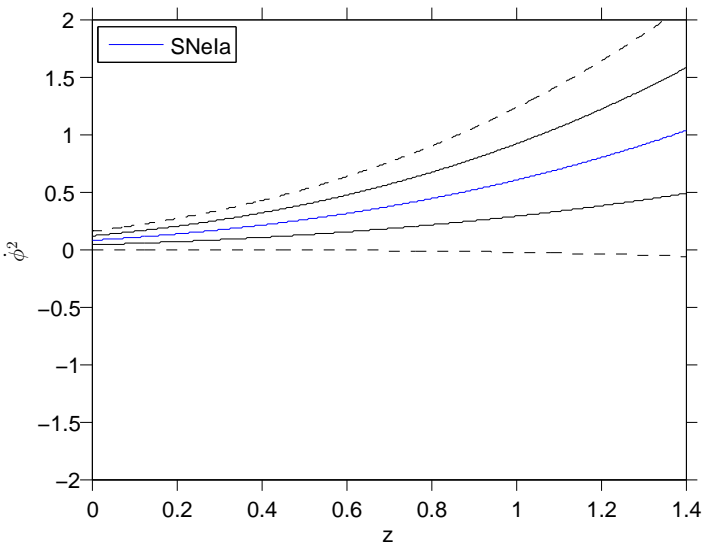

(a)

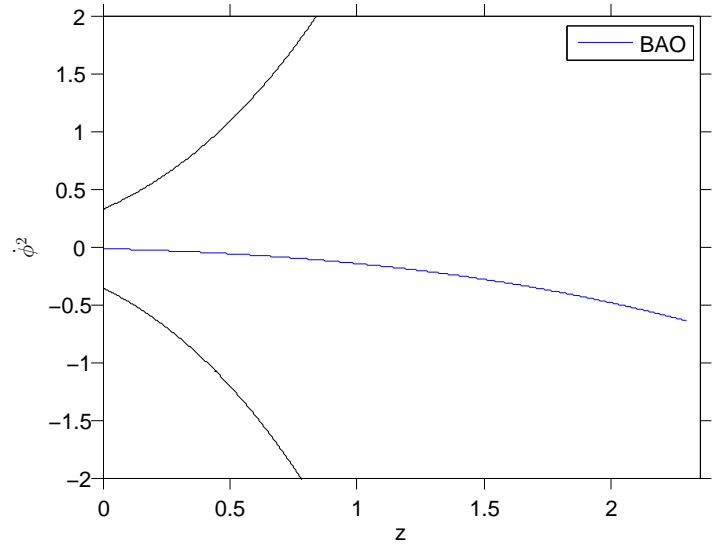

(b)

Figure 2: Solid lines show the reconstructed $\dot{\phi}^{2}$ as a function of redshift with $1 \sigma$ error bars (black curves). In figure (a) the dashed lines corresponds $2 \sigma$ error bars.

$z=0.35$ [43]. Blake et al., gave measurements of $H(z)$ at redshifts $z=0.44,0.6$ and 0.73 by combining measurements of the baryon acoustic peak and Alcock-Paczynski distortion from galaxy clustering in the WiggleZ Dark Energy Survey [44]. Kazin et al., analysed the SDSS-III Baryon Oscillation Spectroscopic Survey (BOSS) CMASS sample of massive galaxies of the ninth data release to measure $H(z)$ at redshift $z=0.57$ [45]. Finally, Busca et al., used the three-dimensional correlation function of the Lyman $\alpha$ forest of high-redshift quasars from the SDSS-III BOSS sample to provide a measurements of $H(z)$ at $z=2.3$ [46]. The data is used to reconstruct $H(z)$ and higher order derivatives simultaneously using GP, and then these functions are used to estimate the dark energy variable. For reconstruction, 
the initial guess for the mean function corresponds to flat $\Lambda$ CDM Universe. There are four hyperprameters in this case: $\sigma_{f}$ and $l$, (from the choice of the covariance function) and $H_{0}$ and $\Omega_{m}$ (from the choice of the mean function). The priors on these parameters are: 0.002 $<\sigma_{f}<1,0.0001<l<2,50<H_{0}<80$ and $0.1<\Omega_{m}<0.6$. The likelihood for this data set can be written as:

$$
L(\boldsymbol{\theta}) \propto \frac{1}{\prod_{i} \sigma_{i}} \exp \left(-\frac{1}{2} \sum_{i}\left(\frac{H_{i}-H\left(z_{i}, \boldsymbol{\theta}\right)}{\sigma_{i}}\right)^{2}\right),
$$

where $\boldsymbol{\theta}$ encapsulated the hyperparameters mentioned above and $\sigma_{i}$ is the error on each $H\left(z_{i}\right)$ as given in the data.

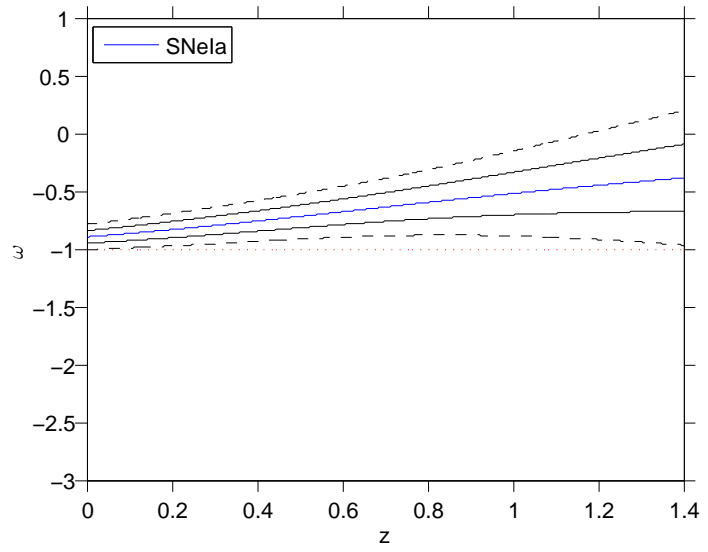

(a)

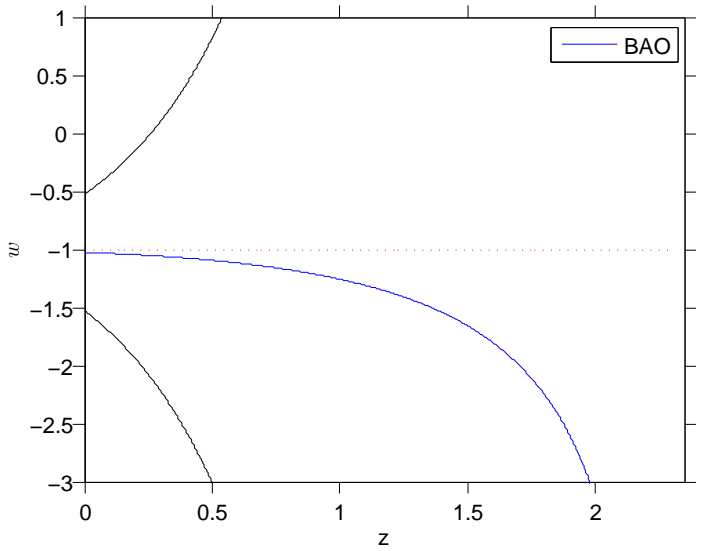

(b)

Figure 3: Solid lines show the reconstructed eos as a function of redshift with $1 \sigma$ error bars (black curves). In figure (a) the dashed lines corresponds $2 \sigma$ error bars.

Supernovae data. We use distance modulus measurements from SNeIa Union2.1 sample, as described in [47], to estimate the luminosity distance $D_{L}(z)$. This sample contains 580 supernovae spanning the redshift range $0.015<z<1.414$. The distance modulus in terms of the redshift, as given in the data is used to estimate the luminosity distance. The relation between the distance modulus $\mu$ and the luminosity distance $D_{L}$ is:

$$
\mu_{B}(z)=m_{B}-M_{B}=5 \log _{10}\left(\frac{D_{L}(z)}{1 M p c}\right)+25,
$$

where $M_{B}$ is the absolute magnitude of the source and $m_{B}$ is the apparent magnitude ( $B$ is for $B$-band). One major source of systematic uncertainty here is the unknown absolute magnitude $M_{B}$. Following Holsclaw et al., we define the distance measure $D(z)=H_{0} D_{L}(z)$ and use it in our analysis (see [26] for details). Here the re-defined distance modulus $\mu^{\prime}$ is

$$
\mu^{\prime}(z)=\mu_{B}(z)+5 \log _{10}\left(H_{0}\right)-25=5 \log _{10}(D(z)) .
$$

The uncertainties in the calibration are accounted for by introducing a shift parameter $\Delta_{\mu}$, in the analysis. The Hubble rate can be written in terms of $D(z)$ as

$$
H(z)=\frac{c H_{0}(1+z)^{2}}{D^{\prime}(z)(1+z)-D(z)} .
$$


We can write equations (2.6) and (2.7) in terms of $D(z)$, and its derivatives as follows:

$$
\begin{aligned}
\frac{8 \pi G}{3 H_{0}^{2}} V(z) & =\frac{c^{2}(1+z)^{4}}{3\left(D^{\prime}(z)(1+z)-D(z)\right)^{2}}\left[1+\frac{D^{\prime \prime}(z)(1+z)^{2}}{D^{\prime}(z)(1+z)-D(z)}\right]-\frac{\Omega_{m}}{2}(1+z)^{3}, \\
\frac{8 \pi G}{3 H_{0}^{2}} \dot{\phi}^{2} & =\frac{2 c^{2}(1+z)^{4}}{\left(D^{\prime}(z)(1+z)-D(z)\right)^{2}}\left[2-\frac{D^{\prime \prime}(z)(1+z)^{2}}{D^{\prime}(z)(1+z)-D(z)}\right]-\Omega_{m}(1+z)^{3} .
\end{aligned}
$$

Other dark energy variables like the pressure, energy density, equation of state etc. can be derived using the above formulae. Similarly the kinematic variables like $\epsilon_{H}$ and the jerk $j$ are derived in terms of $D(z)$ (and its derivatives),

$$
\begin{aligned}
\epsilon_{H} & =2-(1+z)^{2} \frac{D^{\prime \prime}(z)}{D^{\prime}(z)(1+z)-D(z)}, \\
j & =3-\frac{(1+z)^{2}}{\left(D^{\prime}(z)(1+z)-D(z)\right)^{2}}\left[7 D^{\prime \prime}(z)+(1+z) D^{\prime \prime \prime}(z)-3 \frac{(1+z)^{2} D^{\prime \prime}(z)^{2}}{\left(D^{\prime}(z)(1+z)-D(z)\right)}\right] .
\end{aligned}
$$

The GP method is used to reconstruct $D(z)$ and higher order derivatives simultaneously, and then these are used to derive the dark energy variables. For reconstruction, the initial guess for the mean function corresponds to flat $\Lambda$ CDM Universe. There are 5 hyperprameters in this case: $\sigma_{f}$ and $l$, (from the choice of the covariance function) and $\Omega_{m}, \Delta_{\mu}$ and $\tau^{2}$ (from the choice of the mean function and overall scaling of the data). Here $\tau$ accounts for a possible rescaling [26]. The priors on these parameters are: $0.002<\sigma_{f}<1,0.0001<l<1.4,0.1$ $<\Omega_{m}<0.6,-0.5<\Delta_{\mu}<0.5$ and $0.5<\tau^{2}<1$. The likelihood in this case can be written as:

$$
L(\boldsymbol{\theta}) \propto \frac{1}{\tau^{n} \prod_{i} \sigma_{i}}\left[\exp \left(-\frac{1}{2} \sum_{i}\left(\frac{D_{i}-D\left(z_{i}, \boldsymbol{\theta}\right)}{\sigma_{i} \tau}\right)^{2}\right)\right],
$$

where $\boldsymbol{\theta}$ encapsulates the hyperprameters mentioned above.

\section{Results and discussion}

We scan the hyperparameter space using MCMC sampling for both data sets. The probability distribution of the reconstructed function $H(z), H^{\prime}(z)$ and $H^{\prime \prime}(z)$, and $D(z), D^{\prime}(z), D^{\prime \prime}(z)$ and $D^{\prime \prime \prime}(z)$, are weighted by the posterior distribution of the hyperparameters (since our priors are flat, the posterior of the hyperparameters are proportional to the likelihood). For the reconstructed function we obtain a sum of the weighted Gaussian distributions as a result of the marginalization (the integral in the marginalisation of the hyperparameters is approximated as a sum in the discrete case). The mean and covariances for these mixture distributions can be evaluated analytically since they have closed forms (i.e. they too are Gaussian). These weighted mean functions and covariances are then used to derive the dark energy variables and the kinematic variables of cosmic expansion. Note here that variables like the potential, kinetic energy, equation of state etc. have explicit dependence on $\Omega_{m}$. In such cases we use the mean value of $\Omega_{m}$, as obtained from the posterior hyperparameter distribution and include its variance in the overall error.

We also note here that our results may be effected by the choice of the initial mean function $(\Lambda \mathrm{CDM}$ in our case). In this regard, a better choice could be to incorporate an iteration procedure in the analysis, where the posterior found from some preliminary runs are used as mean functions of successive runs (similar to [26]). Another method is to adopt 
some smoothing procedures to generate an initial guess of the mean from say multiple functions [28], and then iterate this to obtain the final results. These methods may give more robust results which are independent of the initial mean function. The estimates of the model

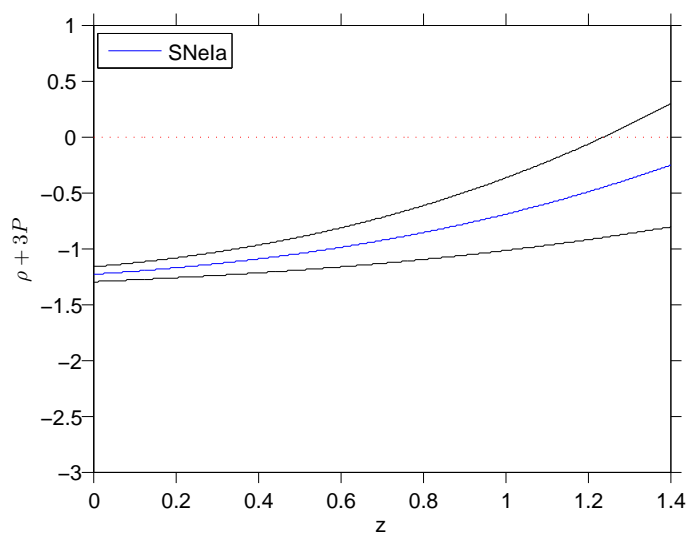

(a)

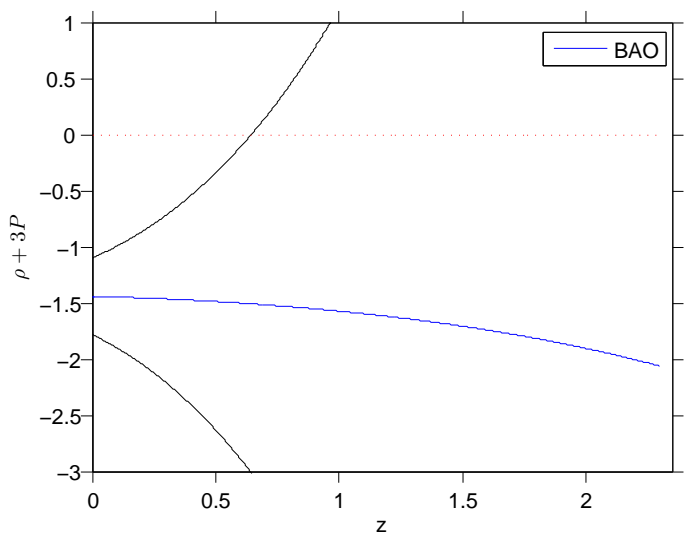

(b)

Figure 4: Solid lines show the reconstructed $\rho+3 P$ as a function of redshift with $1 \sigma$ error bars (black curves). In figure (a) the dashed lines corresponds $2 \sigma$ error bars.

parameters obtained from SNeIa and BAO data are given below.

SNeIa: $\Omega_{m}=0.275 \pm 0.033, \Delta_{\mu}=-0.022 \pm 0.033$ and $\tau^{2}=0.839 \pm 0.114$. The posteriors for $\Omega_{m}$ and $\Delta_{\mu}$, are fairly well peaked. The posterior of $\tau^{2}$ peaks at 0.917. $\sigma_{f}$ and $l$ have nonGaussian posterior and there is high degeneracy between the two. Almost all values in the prior range are allowed. As discussed before it is preferable to marginalize these parameters since optimization may not give robust results.

BAO: $H_{0}=68.789 \pm 6.691$ and $\Omega_{m}=0.3 \pm 0.114$. As before the posteriors for $\sigma_{f}$ and $l$ are non-Gaussian and there is high degeneracy between these parameters.

\subsection{Dark energy dynamics}

Many different kinds of potentials have been described in the literature to explain the evolution of the scalar field [3]. To understand the characteristics of these potentials which can describe the accelerated expansion, is a big challenge. Direct reconstruction of the potential from the observational data is a better approach to understand the physics behind the accelerated expansion of the Universe. There have been many attempts in this direction. Luminosity distances of high redshift supernovae have been used in a parametric setting to constrain the dark energy potential [30, 48]. Parametrized form of effective equation of state of dark energy, deceleration parameter and dark energy density has been used to reconstruct quintessence potential [49]. Huterer and Peiris described the scalar field potentials by using a polynomial series and using PCA obtained constraints on the parameters of the potential [50]. We summarise the results of our analysis of the DE variables below.

(a) We show the reconstruction of the potential $V(z)$, and $\dot{\phi}^{2}$, in Figs. (1) and (2), respectively (in units of critical density). As expected SNeIa data gives better constraints, 


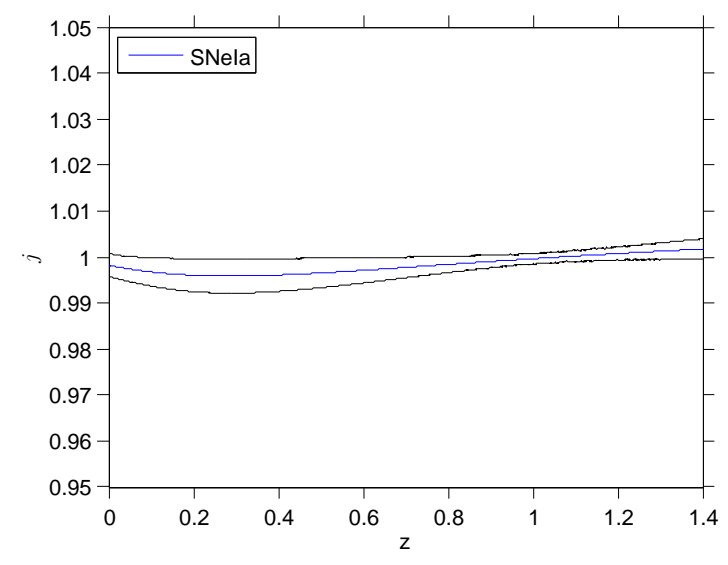

(a)

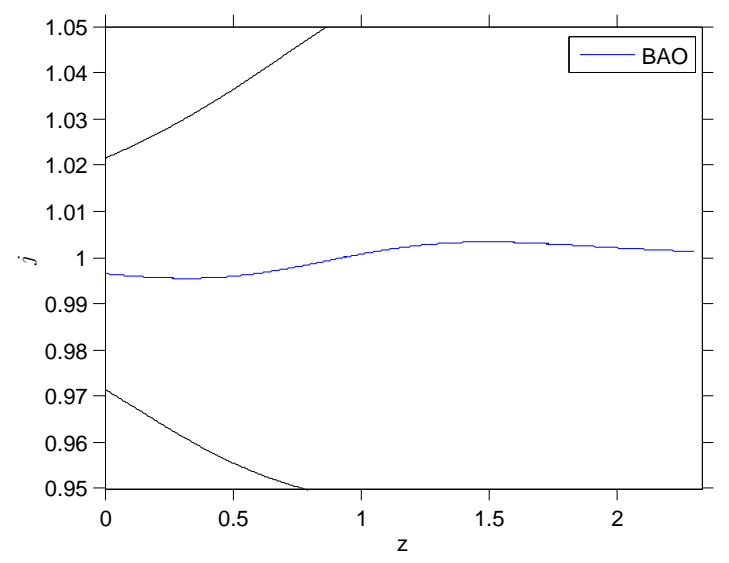

(b)

Figure 5: Solid lines show the reconstructed jerk parameter as a function of redshift with $1 \sigma$ error bars (black curves).

specially at low redshift $(z<0.5)$ compared to BAO data, since the number of data points is much larger.

(b) In case of variables which depend explicitly on $\Omega_{m}\left(V, \dot{\phi}^{2}, \rho+3 P\right.$ and $\left.\omega\right)$, the error budget is effected by the estimated variance of $\Omega_{m}$, which is much larger in BAO data.

(c) In slow roll approximation the kinetic energy of the scalar field approaches zero. This feature is recovered from the SNeIa data at $2 \sigma$ and BAO data at $1 \sigma$ (Fig. 2).

(d) The reconstructed eos $\omega$ (Fig 3) is consistent with the cosmological constant model of dark energy $(\omega=-1)$, at $2 \sigma$ level for SNeIa data and at $1 \sigma$ for BAO data. Our result agrees with the reconstruction of eos by [26] within error bars and we observe their method (of using MCMC within GP modelling to directly reconstruct $\omega$ ) gives tighter constraint on $\omega$.

(e) We also reconstruct the SEC using the SNeIa and BAO observations (Fig 4). We find that it is violated $(\rho+3 P<0)$ for almost the entire redshift range of SNeIa at $1 \sigma$. For BAO data, $\rho+3 P>0$ is allowed at $1 \sigma$ for a large redshift range but we observe that the error bars are too large to derive a conclusion. The NEC is equivalent to $\dot{\phi}^{2} \geq 0$, and we observe that it is satisfied in the entire redshift range of both data sets (Fig 2) within $2 \sigma$ for SNeIa data and within $1 \sigma$ for BAO data. Here too the error bars from BAO are very large.

\subsection{Kinematic variables: $j$ and $\epsilon_{H}$}

Here we observe that the reconstructed error bars on both the kinematic variables are very tight, especially in case of SNeIa data. Here it is worth noting that the majority of the data points is in the near redshift range. So one would expect the error bars to get larger as $z$ increases. This is not evident from the plots of the kinematic variables. As mentioned earlier, the error bars on these variables are obtained through error propagation, where the errors 
on the reconstructed functions, are weighted by the partial derivatives of the variables, with respect to the functions reconstructed $(\partial f / \partial H$ etc.). These weights are redshift dependent and this may be reflected in the overall evolution of the errors on the dynamical variables.

(a) For $\Lambda \mathrm{CDM}$ model $j=1$, and deviation from this value would indicate a possible departure from $\Lambda$ CDM. Barotropic models (where pressure of DE depends strictly on the energy density, i.e. $P=f(\rho)$ ) like generalized Chaplygin gas, satisfy $j \geq 1$ while for quintessence models $j \leq 1$ ([51]). The reconstructed jerk parameter from both data sets agrees well with the $\Lambda$ CDM model (Fig. 5).

(b) The other kinematic variable $\epsilon_{H}$, is related to the deceleration parameter $\left(\epsilon_{H}=1+q\right)$. Hence, the coasting point where $\epsilon_{H}=1$ (i.e. $q=0$ ), gives the transition redshift. SNeIa data and $\mathrm{BAO}$ give $z_{t} \approx 0.54$ and $z_{t} \approx 0.71$ respectively (Fig. 6). Here we note that [28] reconstructed the deceleration parameter $q$, using GP and found constraints using mock as well as real (SNeIa) data. Now, since $\epsilon_{H}$ and $q$ are related by a constant, one can compare these two reconstructions. We observe that, our error bars are tighter than those reported by [28]. In their analysis Arman et al., have accounted for the multiple scale nature of the reconstruction by opting for a smoothing procedure to derive the initial mean function. They use five initial guesses that cross different redshift ranges and cover a wide range of behaviour. In this regard, their error bars may be more robust than ours.

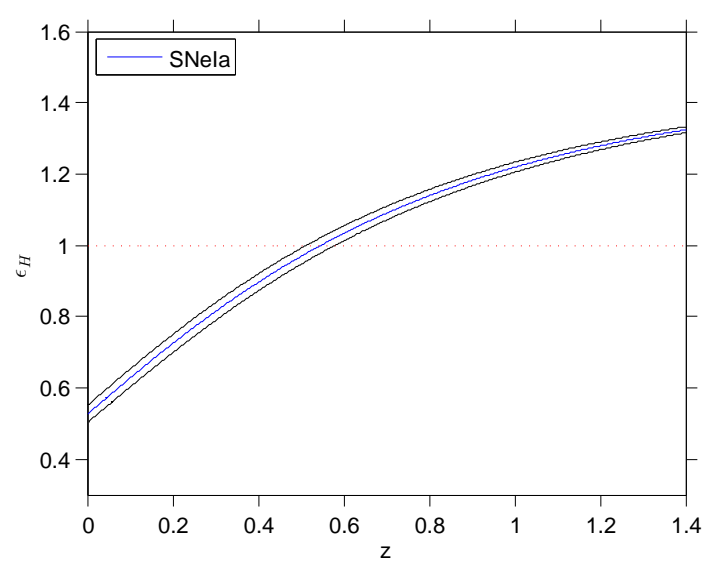

(a)

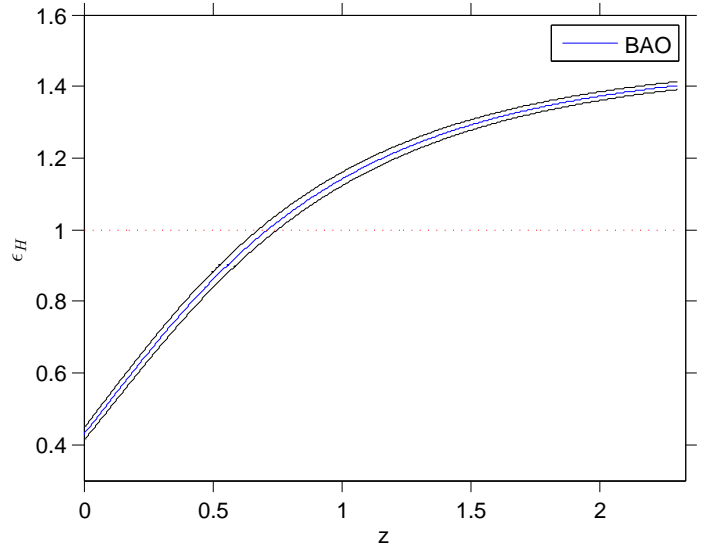

(b)

Figure 6: Solid lines show the reconstructed $\epsilon_{H}$ as a function of redshift with $1 \sigma$ error bars (black curves).

\section{Acknowledgments}

Authors thank the anonymous referee whose valuable suggestions have greatly improved the paper. It is a pleasure to thank Philipp Hennig and David Duvenaud for insightful discussions. One of the author (D.J.) thanks A. Mukherjee and S. Mahajan for providing the facilities to carry out the research. R.N. acknowledges support under CSIR - SRF scheme (Govt. of India). SJ acknowledges support under UGC minor research project (42-1068/2013(SR)). 


\section{References}

[1] Frieman J. et al., Dark Energy and the Accelerating Universe, Ann. Rev. Astron. Astrophys. 46 (2008) 385 [arXiv:0803.0982];

Caldwell R.R. \& Kamionkowski M., The Physics of Cosmic Acceleration, Ann. Rev. Nucl. Part. Sci. 59 (2009) 397 [arXiv:0903.0866].

[2] Sahni V.\& Starobinsky A., Reconstructing Dark Energy, Int. J. Mod. Phys. D 15 (2006) 2105 [arXiv:astro-ph/0610026].

[3] Copeland E.J. et al., Dynamics of dark energy, Int. J. Mod. Phys. D 15 (2006) 1753 [hep-th/0603057].

[4] Supernova Search Team collaboration, Riess A.G. et al., Observational evidence from supernovae for an accelerating universe and a cosmological constant, Astronom. J. 116 (1998) 1009 [astro-ph/9805201];

Supernova Cosmology Project collaboration, Perlmutter S. et al., Measurements of Omega and Lambda from 42 high redshift supernovae, Astrophys. J. 517 (1999) 565 [astro-ph/9812133]; SNLS collaboration, Astier P. et al., The Supernova legacy survey: Measurement of $\Omega_{m}, \Omega_{\Lambda}$ and $w$ from the first year data set, Astron. Astrophys. 447 (2006) 31 [astro-ph/0510447].

[5] Weinberg D.H. et al., Observational Probes of Cosmic Acceleration [arXiv:1201.2434]

[6] Sloan Digital Sky Survey (SDSS): http://www.sdss3.org;

Giant Magellan Telescope (GMT): http://www.gmto.org;

James Webb Space Telescope (JWST): www.jwst.nasa.gov;

Euclid survey: http://sci.esa.int/euclid;

Dark Energy Survey: http://sci.esa.int/euclid;

Large Synoptic Survey Telescope (LSST): http://www.lsst.org;

[7] Weinberg S., The Cosmological Constant Problem, Rev. Mod. Phys. 61, (1989) 1.

[8] Kunz M., The phenomenological approach to modeling the dark energy, Comptes Rendus Physique 13 (2012) 539 [arXiv:1204.5482].

[9] Bassett B. A. et al., The essence of quintessence and the cost of compression, Astrophys. J. Lett. 617 (2004) L1 [astro-ph/0407364].

[10] Vitagliano V. et al., High-redshift cosmography, JCAP 1003 (2010) 005 [arXiv:0911.1249]; Xia J-Q. et al., Cosmography beyond standard candles and rulers, Phys. Rev. D 85 (2012) 043520 [arXiv:1103.0378].

[11] Cai R.G. et al., Probing the dynamical behavior of dark energy, JCAP 04 (2010) 012 [arXiv:1001.2207];

Lu J. et al., Constraints on kinematic models from the latest observational data, Phys. Letts. B 699 (2011) 246 [arXiv:1105.1871];

Xu L. \& Wang Y., Cosmography: Supernovae Union2, Baryon Acoustic Oscillation, Observational Hubble Data and Gamma Ray Bursts, Phys. Letts. B 702 (2011) 114 [arXiv:1009.0963];

Nair R. et al., Cosmokinetics: A joint analysis of Standard Candles, Rulers and Cosmic Clocks, JCAP 01 (2012) 018 [arXiv:1109.4574];

Wang S. et al., Exploring the Latest Union2 SNIa Dataset by Using Model-Independent Parametrization Methods, Phys. Rev. D 83 (2011) 023010 [arXiv:1009.5837];

Giostri R. et al., From cosmic deceleration to acceleration: new constraints from SN Ia and $B A O / C M B, J C A P \mathbf{0 3}$ (2012) 027 [arXiv:1203.3213];

Campo S. del et al., Three thermodynamically-based parameterizations of the deceleration parameter, Phys. Rev. D 86 (2012) 083509 [arXiv:1209.3415];

Sendra I. \& Lazkoz R., SN and BAO constraints on (new) polynomial dark energy 
parametrizations: current results and forecasts, Mon. Not. Roy. Astr. Soc. 422 (2012) 776 [arXiv:1105.4943];

Neben A.R. \& Turner M.S., Beyond $H_{0}$ and $q_{0}$ : Cosmology is no longer just two numbers [arXiv:1209.0480].

[12] Sahni V. et al., Statefinder-A new geometrical diagnostic of dark energy, JETP 77 (2003) 207; Alam U. et al., Exploring the expanding Universe and dark energy using the statefinder diagnostic, Mon. Not. Roy. Astr. Soc. 344 (2003) 1057 [astro-ph/0303009];

Arabsalmani M \& Sahni V. Statender hierarchy: An extended null diagnostic for concordance cosmology, Phys. Rev. D 83 (2011) 043501.

[13] Sahni V. et al., Two new diagnostics of dark energy, Phys. Rev. D 78 (2008) 103502.

[14] Shafieloo A et al., New null diagnostic customized for reconstructing the properties of dark energy from baryon acoustic oscillations data, Phys. Rev. D 86 (2012) 103527.

[15] Wang Y. \& Tegmark M., Uncorrelated measurements of the cosmic expansion history and dark energy from supernovae, Phys. Rev. D 71 (2005) 103513 [astro-ph/0501351].

[16] Zunckel C. \& Trotta R., Reconstructing the history of dark energy using maximum entropy, Mon. Not. Roy. Astr. Soc. 380 (2007) 865 [astro-ph/0702695].

[17] Huterer D. \& Starkman G., Parameterization of dark-energy properties: A Principal-component approach, Phys. Rev. Lett. 90 (2003) 031301 [astro-ph/0207517].

[18] Shapiro C. \& Turner M.S., What do we really know about cosmic acceleration?, Astrophys. J. 649 (2006) 563 [astro-ph/0512586].

[19] Dick J. et al., Reduction of cosmological data for the detection of time-varying dark energy density, JCAP 07 (2006) 001 [astro-ph/0603247];

Nair R. \& Jhingan S., Is dark energy evolving?, JCAP 02 (2013) 049 [arXiv:1212.6644].

[20] Kitching T. D. \& Amara A., Fisher matrix decomposition for dark energy prediction, Mon. Not. Roy. Astr. Soc. 398 (2009) 2134.

[21] Putter R.D. \& Linder E., Being PC: Principal Components and Dark Energy, [arXiv:0812.1794].

[22] Shafieloo A. et al., Smoothing Supernova Data to Reconstruct the Expansion History of the Universe and its Age, Mon. Not. Roy. Astr. Soc. 366 (2006) 1081 [astro-ph/0505329]; Shafieloo A., Model Independent Reconstruction of the Expansion History of the Universe and the Properties of Dark Energy, Mon. Not. Roy. Astr. Soc. 380 (2007) 1573 [astro-ph/0703034]; Shafieloo A. \& Clarkson C., Model independent tests of the standard cosmological model, Phys. Rev. D 81 (2010) 083537 [arXiv:0911.4858].

[23] Shafieloo A. et al., The Crossing Statistic: Dealing with Unknown Errors in the Dispersion of Type Ia Supernovae, JCAP 08 (2011) 017 [arXiv:1006.2141];

Shafieloo A., Crossing Statistic: Bayesian interpretation, model selection and resolving dark energy parametrization problem, JCAP 05 (2012) 024 [arXiv:1202.4808].

[24] Shafieloo A., Crossing statistic: reconstructing the expansion history of the universe, JCAP 08 (2012) 002 [arXiv:1204.1109].

[25] Nesseris S \& Garca-Bellido J., A new perspective on dark energy modeling via genetic algorithms, JCAP 11 (2012) 033 [arXiv:1205.0364].

[26] Holsclaw T et al., Nonparametric Reconstruction of the Dark Energy Equation of State, Phys. Rev. D 82 (2010) 103502 [arXiv:1009.5443].

Holsclaw T et al., Nonparametric Dark Energy Reconstruction from Supernova

Data, Phys. Rev. Lett. 105 (2010) 241302 [arXiv:1011.3079].

Holsclaw T et al., Nonparametric Reconstruction of the Dark Energy Equation of State from Diverse Data Sets, Phys. Rev. D 84 (2011) 083501 [arXiv:1104.2041]. 
[27] Seikel M. et al., Reconstruction of dark energy and expansion dynamics using Gaussian processes, JCAP 06 (2012) 036 [arXiv:1204.2832].

[28] Shafieloo et al., Gaussian process cosmography, Phys. Rev. D 85 (2012) 123530 [arXiv:1204.2272].

[29] Crittenden R. G. et al., Fables of reconstruction: controlling bias in the dark energy equation of state, JCAP 02 (2012) 048 [arXiv:1112.1693].

[30] Saini T.D. et al., Reconstructing the cosmic equation of state from supernova distances, Phys. Rev. Lett. 85 (2000) 6 [astro-ph/9910231].

[31] Li C. et al., Direct reconstruction of the dark energy scalar-field potential, Phys. Rev. D 75 (2007) 103503 [astro-ph/0611093],

Sahlen M., Direct reconstruction of the quintessence potential, Phys. Rev. D 72 (2005) 083511 [astro-ph/0506696],

Simon J. et al., Constraints on the redshift dependence of the dark energy potential,

Phys. Rev. D 71 (2005) 123001 [astro-ph/0412269],

Martinez E.F. \& Verde L., Prospects for constraining the dark energy potential, JCAP 08 (2008) 023 [arXiv:0806.1871],

Vazquez J.A. et al., Reconstruction of the dark energy equation of state, JCAP 09 (2012) 020 [arXiv:1205.0847].

[32] Planck Collaboration, Planck 2013 results. XVI. Cosmological parameters, [arXiv:1303.5076].

[33] S.W. Hawking and G. F. R. Ellis, The Large Scale Structure of Space-Time (Cambridge University Press, Cambridge, England, 1973).

[34] R. Wald, General Relativity (University of Chicago, Chicago, IL, 1984).

[35] S. Carroll, Spacetime and Geometry (Addison-Wesley, Pearson, 2004).

[36] Visser M., General Relativistic Energy Conditions: The Hubble expansion in the epoch of galaxy formation, Phys. Rev. D 56 (1997) 7578 [gr-qc/9705070].

[37] Santos J. et al., Energy conditions and cosmic acceleration Phys. Rev. D 75 (2007) 083523 [astro-ph/0702728];

Lima M.P. et al., Energy condition bounds and their confrontation with supernovae data, Phys. Rev. D 77 (2008) 083518;

Santos J. et al., Energy conditions and supernovae observations Phys. Rev. D 74 (2006) 067301;

Schuecker P. et al., Observational constraints on general relativistic energy conditions, cosmic matter density and dark energy from X-ray clusters of galaxies and type-Ia supernovae, Astron. Astrophys. 402 (2003) 53;

Gong Y. et al., Energy conditions and current acceleration of the universe, Phys. Letts. B 652 (2007) 63;

Wu C-J. et al., Reconstructing the history of energy condition violation from observational data, Astrophys. J. 753 (2012) 2.

[38] Rapetti D. et al., A kinematical approach to dark energy studies, Mon. Not. Roy. Astr. Soc. 375 (2007) 1510 [astro-ph/0605683].

[39] Guimaraes A. C. C. et al., Bayesian Analysis and Constraints on Kinematic Models from Union SNIa, JCAP 10 (2009) 010 [0904.3550].

[40] Zhai Z-X. et al., Reconstruction and constraint of the jerk parameter from OHD and SNe Ia observations, [arXiv:1303.1620].

[41] Rasmussen C. and Williams C., Gaussian Processes for Machine Learning, MIT Press, Cambridge U.S.A. (2006); 
Williams C., Prediction with Gaussian processes: From linear regression to linear prediction and beyond, in Learning in Graphical Models, M.I. Jordan eds., MIT Press, Cambridge U.S.A. (1999);

MacKay D., Information Theory, Inference and Learning Algorithms, Cambridge University Press, Cambridge U.K. (2003).

[42] Gaztanaga E. et al., Clustering of luminous red galaxies IV. Baryon acoustic peak in the line-of-sight direction and a direct measurement of $H(z)$, Mon. Not. Roy. Astr. Soc. 399 (2009) 1663 [arXiv:0807.3551].

[43] Chuang, C. H., \& Wang, Y., Measurements of $H(z)$ and $D_{A}(z)$ from the two-dimensional two-point correlation function of Sloan Digital Sky Survey luminous red galaxies, Mon. Not. Roy. Astr. Soc. 426 (2012) 226 [arXiv:1102.2251].

[44] Blake C. et al., The WiggleZ Dark Energy Survey: Joint measurements of the expansion and growth history at $z<1$, Mon. Not. Roy. Astr. Soc. 425 (2012) 405 [arXiv:1204.3674].

[45] Kazin E. A. et al., The Clustering of Galaxies in the SDSS-III Baryon Oscillation Spectroscopic Survey: Measuring $H(z)$ and $D_{A}(z)$ at $z=0.57$ with Clustering Wedges [arXiv:1303.4391].

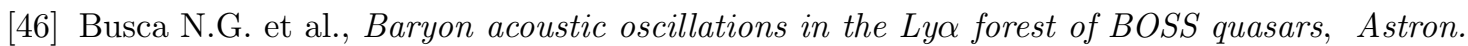
Astrophys. 552 (2013) A96 [arXiv:1211.2616].

[47] Suzuki N. et al., The Hubble Space Telescope Cluster Supernova Survey: V. Improving the Dark Energy Constraints Above $z>1$ and Building an Early-Type-Hosted Supernova Sample, Astrophys. J. 85 (2012) [arXiv:1105.3470].

[48] Chiba T. \& Nakamura T., Feasibility of reconstructing the quintessential potential using type Ia supernova data, Phys. Rev. D 62 (2000) 121301;

Huterer D. \& Turner M.S., Prospects for probing the dark energy via supernova distance measurements, Phys. Rev. D 60 (1999) 081301.

[49] Wang Y. et al., Reconstructing Dark Energy Potentials From Parameterized Deceleration Parameters, Chin. Phys. B. 19 (2010) 019801 [arXiv:1004.3370];

Guo Z-K. et al., Parametrization of quintessence and its potential, Phys. Rev. D 72 (2005) 023504;

Guo Z-K. et al., Parametrizations of the Dark Energy Density and Scalar Potentials, Mod. Phys. Lett. A 22 (2007) 883 [astro-ph/0603109].

[50] Huterer D. \& Peiris H., Dynamical behavior of generic quintessence potentials: Constraints on key dark energy observables Phys. Rev. D 75 (2007) 083503.

[51] Chiba T. \& Nakamura T., The Luminosity Distance, the Equation of State, and the Geometry of the Universe, Prog. Theor. Phys. 100 (1998) 1077 [astro-ph/9808022];

Linder E. V \& Scherrer R. J., Aetherizing Lambda: Barotropic fluids as dark energy, Phys. Rev. D 80 (2009) 023008 [arXiv:0811.2797];

Caldwell R.R \& Kamionkowski M., Expansion, geometry, and gravity, JCAP 09 (2004) 009 [astro-ph/0403003];

Blandford R.D. et al., Cosmokinetics, ASP Conference Series 339 (2005) 27 [astro-ph/0408279]. 